\title{
The Influence of Infrasound Noise from Wind Turbines on EEG Signal Patterns in Humans
}

\author{
C. KASPRZAK* \\ AGH University of Science and Technology, Faculty of Mechanical Engineering and Robotics, \\ Department of Mechanics and Vibroacoustics, Al. A. Mickiewicza 30, 30-059 Krakow
}

\begin{abstract}
The purpose of this paper is to determine the effect of infrasound noise from wind turbines (up to $20 \mathrm{~Hz}$ ) on the changes in the EEG signal patterns in humans. The experimental study was undertaken to investigate the effect of a 20 minutes long infrasound exposure on humans. The acoustic signal was recorded at a distance of 750 meters from the wind turbine and the frequency components above $20 \mathrm{~Hz}$ were then filtered out. Research work undertaken so far to investigate the impacts of wind turbine noise on humans would mostly rely on questionnaire tools and subjective assessment given by respondents. This study focuses on the effects of infrasound noise from wind turbines on variations of EEG signal patterns in an attempt to develop a more objective measure of the infrasound noise impacts. The experimenal study was conducted in a pressure cabin where the EEG procedure was performed. Analysis of the EEG signals reveals the changes between the EEG patterns registered during the three successive stages of the study. The results indicate some changes in EEG signal patterns registered under exposure to wind turbine noise. Moreover, the specific frequency ranges of the EEG signals are found to be altered.
\end{abstract}

DOI: 10.12693/APhysPolA.125.A-20

PACS: 87.50.Y-, 43.28.Dm, 87.19.1r

\section{Introduction}

Wind power has now become one of the most widely exploited and dynamically developing sources of electricity throughout the world. Taking advantage of wind power as a renewable source of energy brings a number of economical, social, environmental, and ecological profits. However, due to the typical size of turbines and their airspace configuration, they can adversely impact the natural environment posing potential hazards such as noise emission, vibrations, non-ionizing radiation effects, emergency situations, the shadow flicker effect, and permanent shade conditions. Turbines may have also a negative effect on the local fauna (particularly birds) as well as the landscape.

In case of wind turbines, both low-frequency noise and audible noise is produced by various aerodynamic noise sources (turbulent layer tearing off from blade edges, boundary layer tearing off, onset of vortex air flows, induction of a boundary vortex, vortices of laminar layer, turbulence of the inflowing air stream) as well as by mechanical noise sources (gearboxes, generators, devices altering the headstock direction, cooling system pumps, ancillary facilities, etc.) [1].

Despite the fact that modern wind turbines operated at daytime generate far less noise than their prototypes, they still appear to strongly affect people. Under certain weather conditions, this noise is transmitted over large distances and exceeds (by about $10-15 \mathrm{~dB}$ ) the noise levels obtained from numerical models [2]. In most cases, this effect can only be sensed in a subjective manner which means that the very presence of wind turbines may

*e-mail: cekasp@agh.edu.pl bring about acoustic or beyond-acoustic annoyance reactions in humans (distraction, irritation). These factors are accounted for in the sound level models and questionnaire tools that were a part of experiments conducted mainly in Holland and Sweden and connected with the level and spectral composition of sound generated by wind turbines emitted over the neighboring areas (residential areas). When addressing the issue, other aspects have to be considered as well: time of the day, atmospheric conditions (wind speed and direction), personal attitude towards wind power generation (ardent supporters and fierce opponents), the actual distance from a wind mill farm or the age of people being interviewed [3-6].

The noise produces negative reactions of the nervous system, affecting such abilities as reading ability, attention, problem solving, and memory. Noise appears to have a negative impact on children at school, mainly because it is impossible to control. It can also lead to elevated levels of stress hormones and increased blood pressure at rest. This unfavorable reaction is stronger in children whose school performance is poor.

Most people do not cope well with the effect of noise exposure, and because of that they run a higher risk of suffering from its harmful effects than it was proved in previous studies [7-9].

In 2001, at the University of Wisconsin-Madison the research workers distributed a questionnaire among the residents who had lived for two years in close proximity of a wind power installation comprising 22 turbines. The results of their investigations showed that $44 \%$ of people living within a distance of 243-402 meters from wind turbines estimated the noise level as an important issue in their households. Similar tendency was observed among $52 \%$ of residents living $804-1600$ meters away from turbines, as well as in the $4 \%$ of those residing $1600-3200$ 
meters from the wind farm. Under certain conditions, wind turbines could still be heard from the distance of $3.2 \mathrm{~km}[10]$.

These findings have been confirmed by Van den Berg, doctor of medicine at Groningen University in Holland, located at the Dutch-German border in the vicinity of a modern wind power plant consisting of 17 turbines with total power of $30 \mathrm{MW}$. Residents who lived $500 \mathrm{~m}$ and further from the turbines reacted strongly to noise pollution, while those living in an approximate distance of $1.9 \mathrm{~km}$ displayed clear signs of annoyance (anger) [2].

In 2005, a survey was carried out among 200 people living $1.2 \mathrm{~km}$ from a French wind mill farm in St. Crepin. $83 \%$ of residents took part in the survey, $27 \%$ found the noise level unbearable at night, $58 \%$ claimed that noises during nights seriously interfered with their night's rest, while $10 \%$ stated that noise in the course of the day was at least distracting, and that was just a six-turbine installation with the rated power of $9 \mathrm{MW}$ [2].

According to the Dutch standards, the noise of wind mill turbines is to be measured based on the average level of exposure Lden (day-evening-night) which is defined in correlation with the wind speed value at the height of the tower [5].

According to Schreurs [4], as long as the infra frequency noise level is kept below $40 \mathrm{~dB}(\mathrm{~A})$, the annoying effects should not be acute and the residents' health should not be strongly affected. For average exposure levels Lden exceeding $45 \mathrm{~dB}(\mathrm{~A})$, the noise is expected to be perceived as irritating and may cause sleep disorders thus affecting human health.

The subjective assessment of sound emitted by wind mill parks was conducted on the basis of surveys collected from residents living nearby wind farms $[5,11]$. The issues addressed included verbal evaluation of noise generated by wind turbine elements, subjective perception of this type noise for different wind conditions as well as the impact of non- acoustic factors (economical benefits, wind mill view, living conditions) on the perceptibility and inconvenience resulting from the noise. The results of surveys carried out in Holland, in which respondents were asked to come up with the most accurate term describing sounds generated by wind power plants located at a distance of $2.5 \mathrm{~km}$ from their permanent residence, revealed that $80 \%$ of respondents described the noise as whistling. This group included both those who sensed certain level of discomfort and respondents who did not complain about the wind mill presence at all [5]. Research efforts aimed at defining the exposure-reaction curve for wind power plants noise both inside and outside houses are summarized in [12].

The purpose of the experiment presented in this paper was to determine the effect of infrasound waves on variations in Delta, Theta, Alpha, SMR, Beta1, and Beta2 waves in humans exposed to infrasound noise in an attempt to give a more objective evaluation of the impacts of infrasound generated by wind turbines.

Papers [13-18] report a statistically significant change in patterns of EEG and ECG signals in humans. Dominant changes were observed in the alpha rhythm during the infrasound exposure.

\section{Methodology}

The test group included subjects (17 men and 18 women) aged 19-23. The examined individuals were volunteers who declared the absence of any medical conditions and were not under the influence of medicines. They had been warned not to drink any stimulating nor intoxicating drinks before the experiment. The acoustic signal was recorded at a distance of 750 meters from a wind turbine, then the frequency components above $20 \mathrm{~Hz}$ were filtered. Audio signals processed this way were applied in the test procedure. The parameters of the applied signal were $S P L=91.6 \mathrm{~dB}(\mathrm{LIN}), S P L=$ $39.2 \mathrm{~dB}(\mathrm{~A})$.

The entire experiment lasted $35 \mathrm{~min}$, the exposure time being $20 \mathrm{~min}$. The acoustic signal, recorded in a wave format, was played from a computer.

After the preparatory activities, the main part of the experiment was conducted consisting in 35 min of constant acquisition of EEG bioelectric signals from the subjects. The study was conducted with the approval of the Local Commission of Bioethics.

The experimental set up comprised: a stimulusgenerating circuit, a Hungarian-type pressure chamber, and the circuit for recording and analysis of EEG signals.

The signal from the WAV-type file was amplified by the electric amplifier ELMUZ 2158M and fed to 6 loudspeakers GDN 30/80/2 installed in the ceiling of the pressure chamber. The acoustic signal (Fig. 1) applied during the experiment was an infrasound with $S P L=$ 91.6 dB(LIN), $S P L=39.2 \mathrm{~dB}(\mathrm{~A})$.

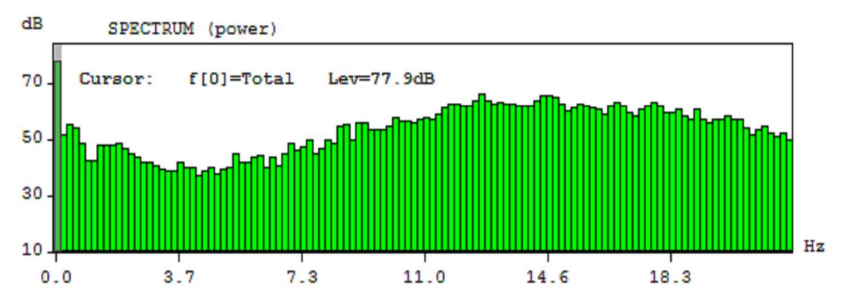

Fig. 1. Acoustic spectrum of the applied infrasound noise.

During the EEG test, the functional currents of a human brain (ranging from several to several hundred microvolts) were registered using electrodes placed on the head skin. The current frequency ranged from $0.5 \mathrm{~Hz}$ to $50 \mathrm{~Hz}$. EEG signals were registered with a 19-channel box of Mitsar-EEG 201 type manufactured by Mistar Company. The EEG cap was fitted in accordance with a standard 10-20 system. The EEG signal was registered at the sampling frequency $256 \mathrm{~Hz}$ using WINEEG application.

The experimental procedure involved three steps: Stage 1- five minutes with no infrasound exposure; Stage $2-20$ minutes of infrasound exposure; Stage 3 
— no infrasound exposure for ten minutes. Experiments were conducted in the pressure chamber and EEG signals were registered throughout the tests using 19 electrodes placed on the participant's head in the 10-20 configuration.

Thus collected EEG data were given a preliminarily treatment to remove the artefacts, then the power spectral density functions were obtained using a 8-second window, $50 \%$ overlapping, and the Haning window. Results obtained at subsequent stages were averaged, the coherence and the EEG signal phase spectrum were determined accordingly. Variations in EEG signal patterns in-between particular stages of the experimental procedure were analyzed. The variance analysis was performed between successive stages of testing for the following EEG rhythms: Delta - 2-4 Hz; Theta - 4-8 Hz; Alpha 8-12 Hz; SMR - 12-15 Hz; Beta1 - 15-22 Hz; Beta2 $-22-32 \mathrm{~Hz}$.

\section{Results}

The EEG patterns were first verified to check their correctness and those that revealed major disturbances were eliminated (1 participant). Moreover, certain errors and artifacts were observed in some channels for 2 subjects. The data from these channels were not analyzed, either.

As regards Delta, Theta, Alpha, SMR, Beta1, and Beta2 waves, the assumption was made that good correspondence should be found between their averaged amplitudes and the normal distribution pattern. This hypothesis was found to hold good, hence the distribution of the variables should be regarded as normal $(p>0.05)$. The multivariate variance analysis MANOVA was performed.

Figures 2, 3 and 4 shows distributions of Delta, Theta, Alpha, SMR, Beta1, and Beta2 rhythm amplitudes registered in various stages of research, revealing a decrease in the amplitude of the Alpha rhythm during Stage 2 and return to the baseline after the infrasound exposure was over. A similar patterns was observed in the case of the Theta rhythm.

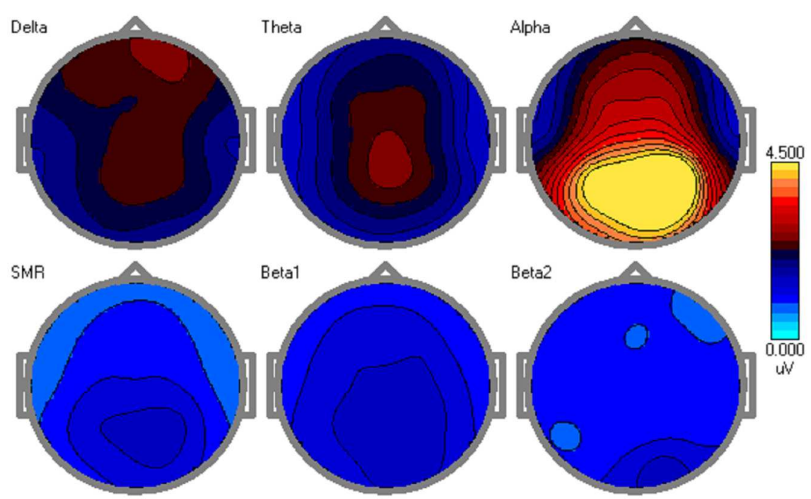

Fig. 2. Distribution amplitude: Delta, Theta, Alpha, SMR, Beta1, and Beta2 in Stage 1.

Quantitative changes in amplitude in different EEG rhythms were investigated in the next stage of the analysis. The multivariate variance analysis (MANOVA) was
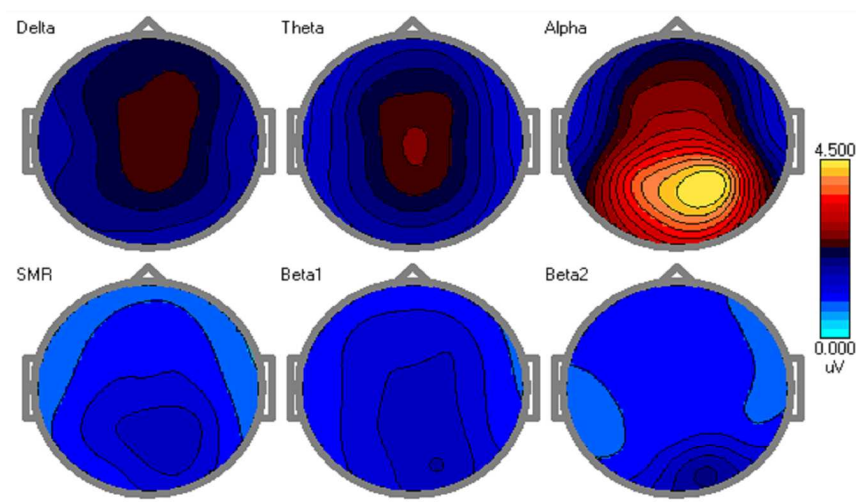

Fig. 3. Distribution amplitude: Delta, Theta, Alpha, SMR, Beta1, and Beta2 in Stage 2.
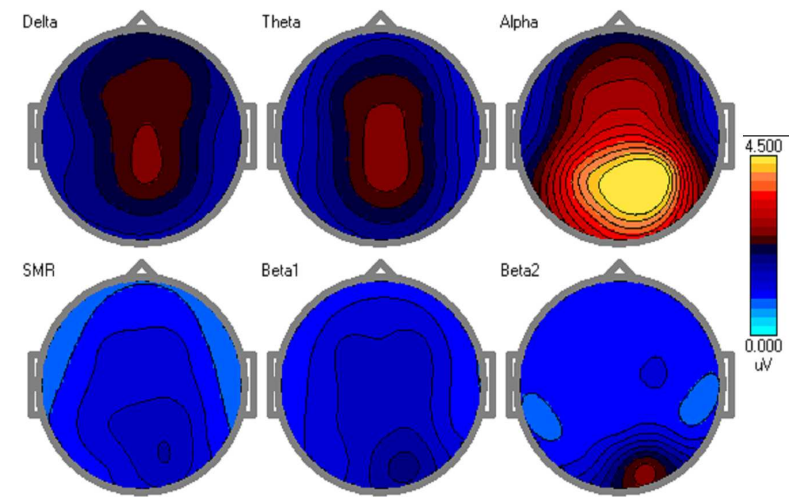

Fig. 4. Distribution amplitude: Delta, Theta, Alpha, SMR, Beta1, and Beta2 in Stage 3.

performed for the $3 \times 19$ configuration (3 stages $\mathrm{x} 19$ EEG electrodes). The ANOVA analysis data for particular EEG rhythms are summarized in Table I, revealing no statistically changes between successive stages of the experiment. The univariate ANOVA analysis for a single EEG electrode reveals no statistically significant changes, either.

\section{TABLE}

Significance of differences of rhythm power between particular stages of the experiment, Wilks test. MANOVA test statistics: Value - value of Wilk test, $F-F$-ratio, $\mathrm{EF}$ - effect degrees of freedom, ER - error degrees of freedom, $p-p$-value

\begin{tabular}{c|c|c|c|c|c}
\hline \hline Rhythm & Value & $F$ & EF & ER & $p$ \\
\hline Delta & 0.753 & 0.6722 & 38 & 168 & 0.92 \\
Theta & 0.789 & 0.5539 & 38 & 168 & 0.98 \\
Alpha & 0.892 & 0.2584 & 38 & 168 & 0.99 \\
SMR & 0.837 & 0.4096 & 38 & 168 & 0.99 \\
Beta1 & 0.758 & 0.6547 & 38 & 168 & 0.93 \\
Beta2 & 0.787 & 0.5615 & 38 & 168 & 0.98
\end{tabular}

Figure 5 shows the amplitude of the qualitative changes between Delta, Theta, Alpha, SMR, Beta1 and Beta2 waves registered at Stages 2 and 1. 


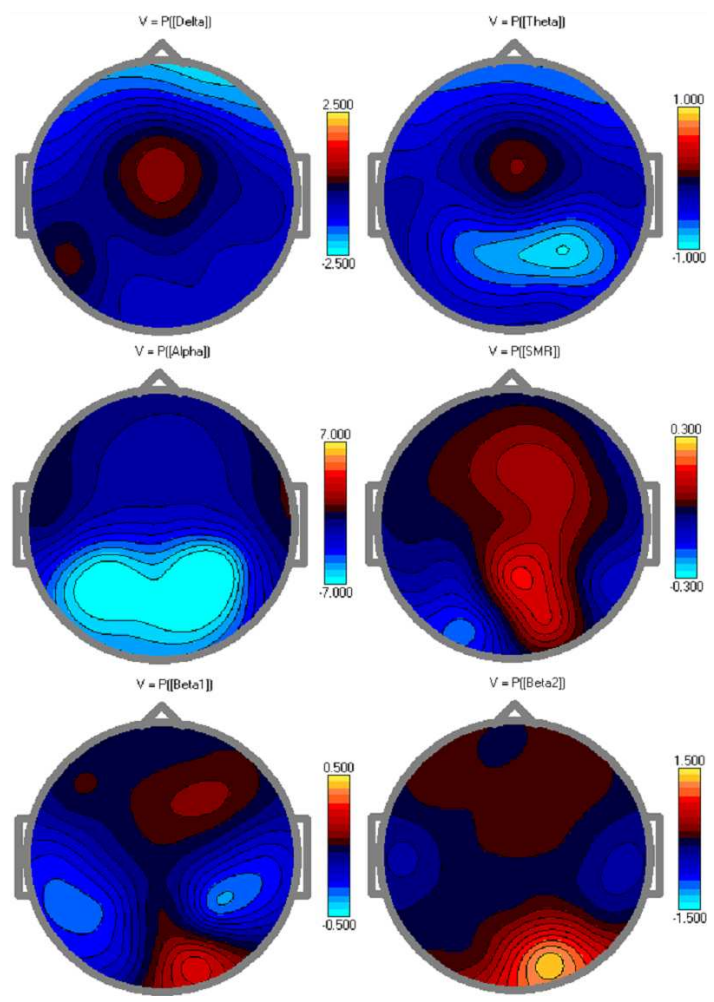

Fig. 5. Amplitude differences of Delta, Theta, Alpha, SMR, Beta1, and Beta2 waves between Stages 2 and 1.

The Alpha wave amplitude is reduced during the infrasound exposure, which is best revealed in the occipital region. The SMR amplitude tends to increase during the infrasound exposure.

\section{Conclusions}

The purpose of this paper is to determine the effect of infrasound noise generated by wind turbines (up to 20 $\mathrm{Hz}$ ) on the changes in the EEG signal patterns in humans in an attempt to give a more objective evaluation of the infrasound annoyance. Previous studies [13-18] that explored the impact of infrasound on humans have shown statistically significant changes in EEG signal patterns such as some variations in the alpha rhythm and the occurrence of the frequency driving response during the infrasound exposure.
Studies on the impact of infrasound noise from wind farms revealed no significant changes in the amplitude of selected EEG rhythms. The Alpha wave amplitude was found to decrease during the infrasound exposure but this result was not statistically significant.

\section{Acknowledgments}

This study is a part of the N N501 247740 research project supported by the National Science Centre.

\section{References}

[1] S. Oerlemans, P. Sijtsma, L. Méndez, J. Sound Vibr 299, 869 (2007).

[2] G.P. van den Berg, J. Sound Vibr. 277, 955 (2004).

[3] M.V. Lowson, Proc. Internoise 96, 479 (1996).

[4] E. Schreurs, J. Jabben, E. Verhejien, Proc. Euronoise 2009, 3975 (2009).

[5] G.P. van den Berg, Proc. Euronoise 2009, 3965 (2009).

[6] E. Pedersen, G.P. van den Berg, R. Bakker, J. Bouma, J. Acoust. Soc. Am. 126, 634 (2009).

[7] B. Berglund, P. Hassmén, R.F. Soames, J. Acoust. Soc. Am. 5, 2985 (1996).

[8] M. Alves-Pereira, Aviation, Space Environmental Medicine 70, 7 (1999).

[9] M. Branco, M. Alves-Pereira, Noise Health 23, 3 (2004).

[10] D.E. Kabes, C. Smith, Excerpts from the final report of the Township of Lincoln Wind Turbine, Agricultural Resource Center, Madison 2001.

[11] E. Pedersen, Human response to wind turbine noise - perception, annoyance and moderating factors, Sahlgrenska Academy, Göteborg 2007.

[12] S.A. Janssen, A.R. Eisses, E. Pedersen, Euronoise 2009, 1472 (2009).

[13] C. Kasprzak, Acta Phys. Pol. A 118, 87 (2010).

[14] C. Kasprzak, Acta Bio-Optica Inform. Med. 15, 390 (2009).

[15] Z. Damijan, C. Kasprzak, R. Panuszka, J. Acoust. Soc. Am. 115, 2388 (2004).

[16] C. Kasprzak, Acta Phys. Pol. A 121, 61 (2012).

[17] C. Kasprzak, Acta Phys. Pol. A 119, 986 (2011).

[18] C. Kasprzak, Acta Phys. Pol. A 123, 980 (2013). 